\title{
A Dual-Synchrony Chaotic Communication Scheme
}

\author{
Noah F. Reddell ${ }^{1,2}$, Erik M. Bollt ${ }^{3}$, Thad B. Welch ${ }^{2}$ \\ ${ }^{1}$ Stanford University, Department of Electrical Engineering \\ Stanford, CA \\ ${ }^{2}$ US Naval Academy, Electrical Engineering Department \\ Annapolis, MD \\ ${ }^{3}$ Department of Mathematics and Computer Science \\ Clarkson Unviversity \\ Potsdam, NY
}

February 21, 2005

\begin{abstract}
We demonstrate here a new dual receiver synchronization scheme that works because we are able to store samples over an entire bit period and then perform an intelligent comparison by methods we introduce. We discuss advantages for message camouflage of transmissions near the noise floor. Our results show better bit error probability performance in comparison to previously published methods. This represents a significantly different and promising new way to use chaotic transmitters to advantageously transmit designed signals.
\end{abstract}

\section{Introduction}

Chaotic systems are a class of aperiodic deterministic dynamical systems that are sensitive to slight variations in initial condition. A system's sensitive dependence to initial condition results in the problem that the behavior of the system cannot be predicted for a significant period into the future. The state of a system for the next instant is completely deterministic, but in the long run it cannot be calculated with any degree of accuracy.

These systems then produce random-like behavior due to their unpredictability and relatively wide frequency content. We have looked at both the frequency domain and time domain properties of chaotic systems and find that using them for a message carrier could offer several advantages over traditional modulation schemes such as amplitude modulation and frequency modulation. Our goal is to design a system that can camouflage a transmission near the noise floor.

It may seem strange to attempt communication using a chaotic carrier since the state of a chaotic system cannot be accurately predicted. However, a number of chaotic communication schemes have been proven possible and useful based on the property of self-synchronization [1], [2].

Some chaotic systems can be synchronized with an identical system by allowing for some influence between the two. Both systems will remain chaotic, but one locks to the other. Once synchronization has been achieved, information can be sent. A transmitter's output is modified in some way by a message. Since the receiver follows what the transmitter's state should be, it can detect the modification caused by a message and thus extract the information from the chaotic signal. Meanwhile, the transmission will hopefully continue to look like noise to an outside observer. 
In this paper, we will present a new dual synchronizer communication scheme, in which bits will be defined based on one of two oscillators at the receivers end synchronizing to a transmitters signal. We will discuss implementing this scheme within DSP hardware. This paper is an extension of our earlier work in [7].

\section{Synchronized Chaos}

We consider the famous Lorenz System:

$$
\begin{aligned}
\dot{x} & =\sigma(y-x), \\
\dot{y} & =r x-y-x z, \\
\dot{z} & =x y-b z .
\end{aligned}
$$

The parameters $\sigma, r$, and $b$ have been removed from their original context in Lorenz's convection process but they are still significant for our purposes. It turns out that the Lorenz system given above has a dynamic range that is impractical for the Digital-to-Analog and Analog-to-Digital converters (CODECs) on our DSPs. Additionally, the system evolves at a rate that is impractical for the sampling rate of the CODECs. For these reasons, we will use a magnitude and time scaling change of variables. Scaling magnitude by $\frac{1}{A}$ allows the $x$ term to be sent to the Digital-to-Analog converter without saturation. A time scale of $T_{S}$ allows efficient use of available CODEC bandwidth. These terms will need to be adjusted based on the particular parameters chosen and the time scaling will be dependent on the step size of the differential equation solver.

The uniform scaling is given by the substitution:

$$
u=\frac{x}{A}, v=\frac{y}{A}, w=\frac{z}{A} .
$$

Thus, the scaled drive system (transmitter) is:

$$
\begin{aligned}
\dot{u} & =T_{S} \sigma(v-u), \\
\dot{v} & =T_{S}(r u-v-A u w), \\
\dot{w} & =T_{S}(A u v-b w) .
\end{aligned}
$$

\subsection{The Dual Synchronizer System: Drive - Response Coupling based on Param- eter Set Match or Mismatch}

In our discrete scheme, we further an idea initiated by Cuomo, et al. [3]. They sent a binary message by adjusting the $b$ parameter of the drive system. This adjustment slightly upsets the synchronization between the drive and response systems. The presence or absence of error at the response system could then be used to determine the message bit.

Our new dual synchronizing response system is as follows. We run two response systems in the receiver DSP. One response system parameter set corresponds to a one-bit and the other corresponds to a zero-bit. Both systems attempt to synchronize with the drive system over the entire bit period. Then, the errors experienced by each response system are compared. The system with less error determines the received bit and both response system states are updated to reflect the better match. By taking advantage of the abilities of DSP hardware, we achieve better performance than a discrete version of the system in [3].

Figure 1 shows four cases of two drive systems and two response systems . The drive system chooses parameter set A or B based on the message bit. The plots show the drive system and how 
the response systems (one using set A and one using set B) respond. We desire that a matched set of parameters between the transmitter and receiver causes a quick and tight coupling while a mismatched set leads to a large error.

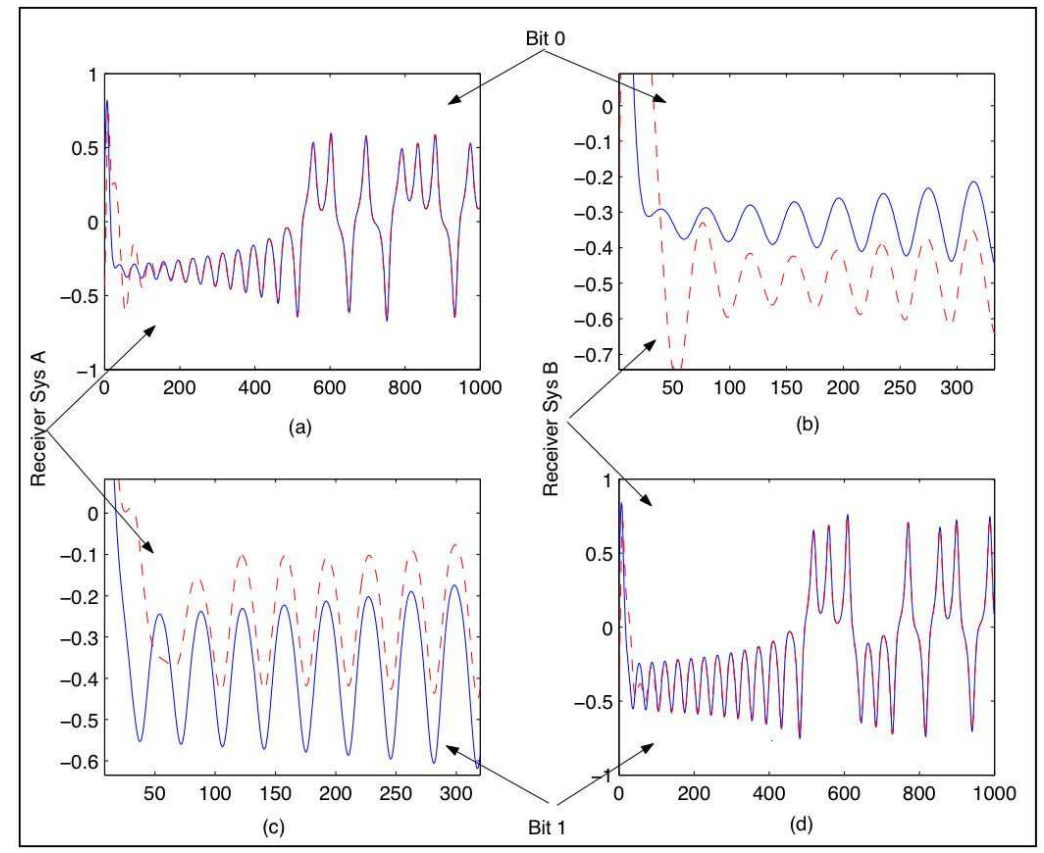

Figure 1: All possible combinations of bit sent and receiver system, plotted as voltage vs. sample number. (a), transmitter and receiver use parameter set A (b), transmitter uses set A, receiver uses set B, (c), Transmitter uses set B, receiver uses set A (d), transmitter and receiver use parameter set B.

For effectiveness of this system, we must now address questions of stability, and instability, to try to make the system bearing the desired bit to be most stable, while the other system is unstable. So first we discuss the error system.

Coupling is achieved by sharing the $u$ term from the drive system with the response system. Notice in eqn. (4) that $u$ takes the place of $u_{r}$ in the equations for $\dot{v}_{r}$ and $\dot{w}_{r}$. The variable $u$ is the influence signal. We maintain the same influence configuration as used by Cuomo, et al. and simplify the problem by letting $\sigma$ and $r$ be the same in the response systems as the drive system. The transmitter alters the drive system parameter $b$ based on a message bit. Parameter $b_{r}$ represents the counterpart parameter in the response system. This $b_{r}$ will either be identical or mismatched.

The response system (receiver) is:

$$
\begin{aligned}
\dot{u}_{r} & =T_{S} \sigma\left(v_{r}-u_{r}\right) \\
\dot{v}_{r} & =T_{S}\left(r u-v_{r}-A u w_{r}\right), \\
\dot{w}_{r} & =T_{S}\left(A u v_{r}-b_{r} w_{r}\right) .
\end{aligned}
$$

Error terms are used to evaluate coupling:

$$
\begin{aligned}
e_{u} & =\left(u-u_{r}\right), \\
e_{v} & =\left(v-v_{r}\right), \\
e_{w} & =\left(w-w_{r}\right) .
\end{aligned}
$$


Taking the derivative with respect to time yields

$$
\begin{aligned}
\dot{e}_{u} & =\left(\dot{u}-\dot{u}_{r}\right), \\
& =T_{S} \sigma(v-u)-T_{S} \sigma\left(v_{r}-u_{r}\right), \\
& =T_{S} \sigma\left(e_{v}-e_{u}\right) . \\
\dot{e}_{v} & =\left(\dot{v}-\dot{v}_{r}\right), \\
& =T_{S}\left(r u-v-A u w-r u+v_{r}+A u w_{r}\right), \\
& =T_{S}\left(-e_{v}-A u e_{w}\right) . \\
\dot{e}_{w} & =\left(\dot{w}-\dot{w}_{r}\right), \\
& =T_{S}\left(A u v-b w-A u v_{r}+b_{r} w_{r}\right), \\
& =T_{S}\left(A u e_{v}-b w+b_{r} w_{r}\right) .
\end{aligned}
$$

\subsection{Lyapunov Function Analysis}

If we can find a Lyapunov function for the error system above, we can show that it approaches zero over time, and thus the two Lorenz systems synchronize [4]. Lyapunov functions generalize the idea of potential energy. Again we follow Cuomo's lead and use his Lyapunov function as the basis for ours [3].

$$
E\left(e_{u}, e_{v}, e_{w}\right)=\frac{1}{2}\left(\frac{1}{\sigma} e_{u}^{2}+e_{v}^{2}+e_{w}^{2}\right) .
$$

To show synchronization, we want to find that the function $E\left(e_{u}, e_{v}, e_{w}\right)$ has a long-term negative slope and so error decreases. Taking the derivative with respect to time:

$$
\begin{aligned}
\frac{d E}{d t}= & \frac{\partial E}{\partial e_{u}} \cdot \frac{\partial e_{u}}{\partial t}+\frac{\partial E}{\partial e_{v}} \cdot \frac{\partial e_{v}}{\partial t}+\frac{\partial E}{\partial e_{w}} \cdot \frac{\partial e_{w}}{\partial t} \\
= & \frac{e_{u} \dot{e}_{u}}{\sigma}+e_{v} \dot{e}_{v}+e_{w} \dot{e}_{w} \\
= & T_{S}\left(e_{u} e_{v}-e_{u}^{2}-e_{v}^{2}-A u e_{v} e_{w}\right. \\
& \left.+ \text { Aueve }_{w}-e_{w}\left(b w-b_{r} w_{r}\right)\right) .
\end{aligned}
$$

If $b=b_{r}$ (Parameter Set Match) then,

$$
\begin{aligned}
\frac{d E}{d t} & =T_{S}\left(e_{u} e_{v}-e_{u}^{2}-e_{v}^{2}-b e_{w}^{2}\right) \\
& =T_{S}\left(-\left(e_{u}-\frac{1}{2} e_{v}\right)^{2}-\frac{3}{4} e_{v}^{2}-b e_{w}^{2}\right)
\end{aligned}
$$

Since $E$ is positive definite and $\dot{E}$ is negative definite with $T_{S}>0$, Lyapunov's theorem implies e(t) approaches 0 as $\mathrm{t} \rightarrow \infty$. Synchronization will therefore occur. For our dual synchronizer system to work, it is necessary that reasonable degree of convergence occurs within one bit period, so that there will be a positively discernible read that one or the other bit receiver systems indicated the signal. The Lyapunov function analysis does not indicate how fast it occurs, but experimentation shows it to be fast enough to achieve a working system.

If $b \neq b_{r}$ (Parameter Set Mismatch) then,

$$
\frac{d E}{d t}=T_{S}\left(e_{u} e_{v}-e_{u}^{2}-e_{v}^{2}-e_{w}\left(b w-b_{r} w_{r}\right)\right)
$$

The derivative above is inconclusive. Likewise, mismatch of the other two parameters leads to a similar conclusion. In Section 6, we will discuss optimally choosing parameter values to improve BER based on allowing mismatch in the $\sigma$ and $r$ parameters, using Lyapunov exponent analysis. 


\section{Development of the Discrete Carrier}

The differential equations described above are continuous systems and must be modified to run our discrete hardware. This can be done by using a differential equation solving algorithm. We have chosen to use the Runge-Kutta 4-5 algorithm because it yields accurate results relative to its processing requirements [5]. The chief issue we have faced when transforming the continuous systems to a discrete environment is that of step size.

The transmission system takes one step via the RK-45 algorithm every time the digital-to-analog converter interrupt service routine is called. This rate is fixed at $48 \mathrm{kHz}$ by our CODECs. Instead, the system can be sped up or slowed down by adjusting the RK-45 step size or by adjusting the time scale $T_{S}$, which are a related pair. To most effectively utilize the available bandwidth of the CODEC without aliasing, we have found that the limiting factor is the step size- $T_{S}$ pair. This is because taking a step that is too big causes the RK-45 algorithm to fail and the discrete system does not emulate its continuous model. In the end, we have a system that is sampled at a rate greater than what Nyquist would require. We have not yet experimented with discarding unnecessary samples.

\section{Implementation}

In Section 6, we will discuss optimizing BER with respect to parameter values. For now, we have chosen the two values for $b$ to be $b(0)=2.0$ and $b(1)=6.5$.

Figure 2 shows a single bit window used in our dual synchronizing receiver scheme. This particular window is 100 samples long. The influence signal from the transmitter is affected by additive Gaussian noise and the two receiver versions attempt to synchronize to the influence signal. The error squared is plotted below. The sum of squares of the error for both receiver systems is used to determine the best match with the influence signal. This comparison yields the received bit.

To illustrate the effects of noise on our scheme, Figure 2 shows a single bit window used in our dual synchronizing receiver scheme. The influence signal from the transmitter is affected by additive white Gaussian noise and the two response systems in the receiver attempt to synchronize to the noise influence signal. The sum of squares of the error for both receiver systems is used to determine the best match with the influence signal. For tis case, SysB represented by the open circles is the better match. This system would then determine the received bit, and the other response system, sysA would be reset to match the state of sysB before the next bit period.

Because the DSP hardware gives us the ability to compare two receiver versions against the received signal, we do not have to worry about completely destroying the synchronization by a parameter mismatch which is too large. The parameter matched version will reset the state of both response systems after the bit period. As a result of our system's ability to move forward with the best fit system, we are able to maintain synchronization with an aggressive parameter mismatch. For now, we have chosen the two values for $b$ to be $b(0)=2.0$ and $b(1)=6.5$, but we discuss how to make such a choice in Section 6.

\section{$5 \quad$ Bit Energy Rate}

A bit error rate is considered to be a fundamental way to evaluate a communication systems performance in the presence of noise. These curves relate bit-error probability to the ratio of bit energy to noise power spectral density $\frac{E_{b}}{N_{0}}$. For just a few specific schemes, such as the binary phase-shift key (BPSK) there are known closed form expressions for the bit-error rate. In general, however, we must resort to standard techniques of Monte-Carlo sampling to simulate the transmitter, receiver and the 


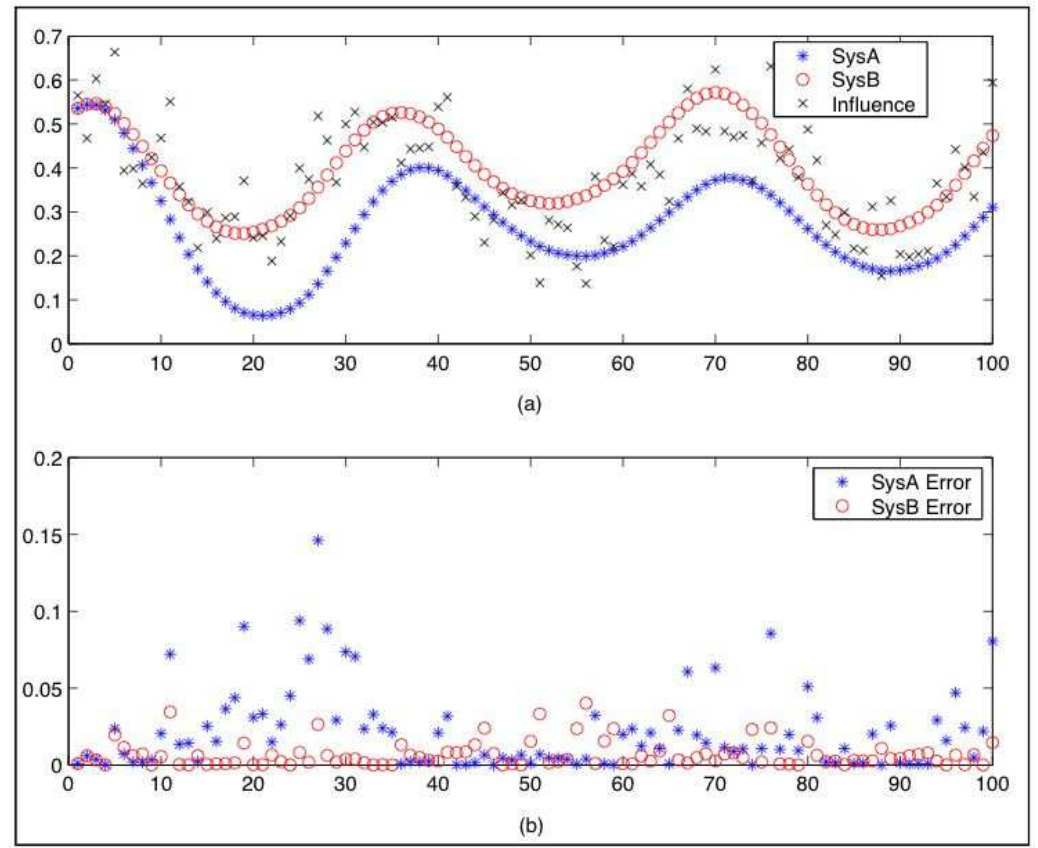

Figure 2: Receiver evaluation of a particular bit, (a) Influence signal and attempts to synchronize by both receiver systems (Volts vs. Sample Number), (b) Error ${ }^{2}$ between both receivers and the influence signal (Volts ${ }^{2}$ vs. Sample Number.)

communications channel to approximate the curves BER, as we do here and shown in Figure 3. The first set of simulations highlight the performance of our dual synchronizing receiver scheme with parameter sets that have been used in another published work. Coumo, et. al. present a communication scheme based in [3]. In their single parameter modulation scheme, the $\beta$ term is the only parameter changed based on the message bit to be transmitted. The open squares in Figure 3 show the performance of our system when modulating only the $\beta$ parameter based on a message bit. $\beta(0)=4.0$, and $\beta(1)=4.4$, and the other two parameters constant $\sigma=16.0, \rho=45.6$.

Using the memory of our discrete processor based system in our dual synchronizing receiver scheme allows a more substantial parameter set mismatch between a one bit and a zero bit. By running two receivers, one for each parameter set, the transmitter state and the receiver state will always reasonably match at the end of any bit period. The asterisks in Figure 3 show the performance of our system using a more substantial modulation of $\beta$. $(\beta=4.0$, and $\beta(1)=4.4)$. The other two parameters are constants $\sigma=16.0$, and $\rho=45.6$.

The open circles in Figure 3 show the bit error probability results by Carrol and Pecora [6] for comparison. We contend below that our method is very strong for camouflage and here we show a stronger BER curve.

The bit period is a crucial factor for bit error probability simulation because it affects how much time the two receivers have $t$ synchronize to or diverge from the influence signal. This also directly affects system data rate. Our sample count for these simulations is 100 samples per bit. At 48,000 samples per second, the bit period is $T=2.08 \mathrm{msec}$. For comparison, the bit period for the open circles is reported to be 200 sec.

We have discovered, a unique feature/problem characteristic of the dynamical system itself. While trying to ascertain the cause of bit errors, we found that they are largely due to characteristics of the system itself for relatively large $\frac{E_{b}}{N_{0}}$. For basic transmission schemes like BPSK, the energy in a bit 


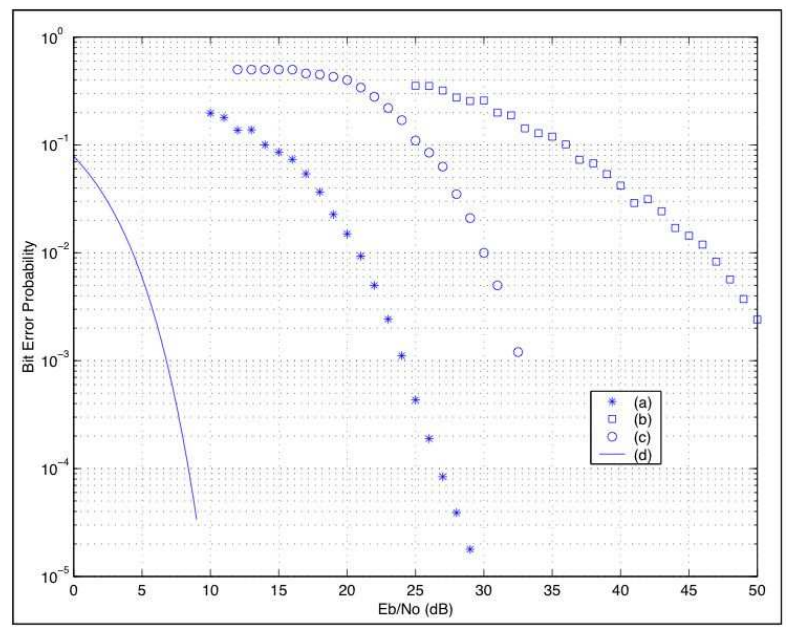

Figure 3: Bit error probability as a function of the ratio of energy per bit $E_{b}$ to noise power spectral density $N_{0}$ for several communications schemes. (a), the asterisks show the performance of our discrete system using parameter modulation techniques with a good parameter set (b), the open squares show the performance of our discrete system using the more conservative parameter mismatch used in [3] (c), the open circles show the performance of the multiple attractor system in [6] (d), the solid line shows results for baseband BPSK for comparison .

is always the same and errors occur when the noise energy is large. This is not true for this chaotic scheme. It turns out that the Lorenz system occasionally goes into regions where the power of $u$ is significantly less than its average. When the bit window corresponds to these regions, the energy in those bits is smaller than expected. For relatively large values of $\frac{E_{b}}{N_{0}}$ (in $\mathrm{dB}$ ) bit errors are largely due to characteristics of the chaotic system instead of noise. For basic transmission schemes like BPSK, the energy in a bit is always the same and errors occur when the noise energy is large. This is not true for our chaotic scheme. Recall that we used average bit energy $E_{b, A v g}$ for our calculations of noise power spectral density. Figure 4 shows a histogram of bit energy and noise energy for 200 bit error observations. This system was running with an average $\frac{E_{b}}{N_{0}}$ of $29 \mathrm{~dB}$. The histograms indicate that the majority of errors occur when the bit energy is small rather than when the noise energy is large.

\section{Parameter Space Search for Improved Bit Energy}

For our dual synchronizing receiver system, we wish to choose the system parameters, $\sigma, \rho$ and $\beta$ of the Lyaponov system so as to optimize the system performance. What we wish is that synchronization will occur rapidly, and desynchronization will also occur rapidly. The Lyapunov function analysis alone of the previous section is not capable of such issues. We consider the Lyapunov exponents [8] as parameter search for "best performance," to be defined below . Lyapunov exponents measure average error growth between two nearby solutions of a system [8]. A positive exponent indicates that nearby solutions diverge in the long run. Boundedness and a positive Lyapunov exponents is one popular definition of chaos.

The full problem would be to perform a six parameter search, in the two sets of $\rho, \sigma, \beta$ for "best" Lyapunov exponents sets. Due to the computational cost of such a high-dimensional search, and the lack of any kind continuity of exponents to help automate the search, we have restricted to a three dimensional search based on fixing one parameter set in order to maximize $E_{d} i f f$, where we have found that the performance of the system is essentially based on the difference between the energy of 


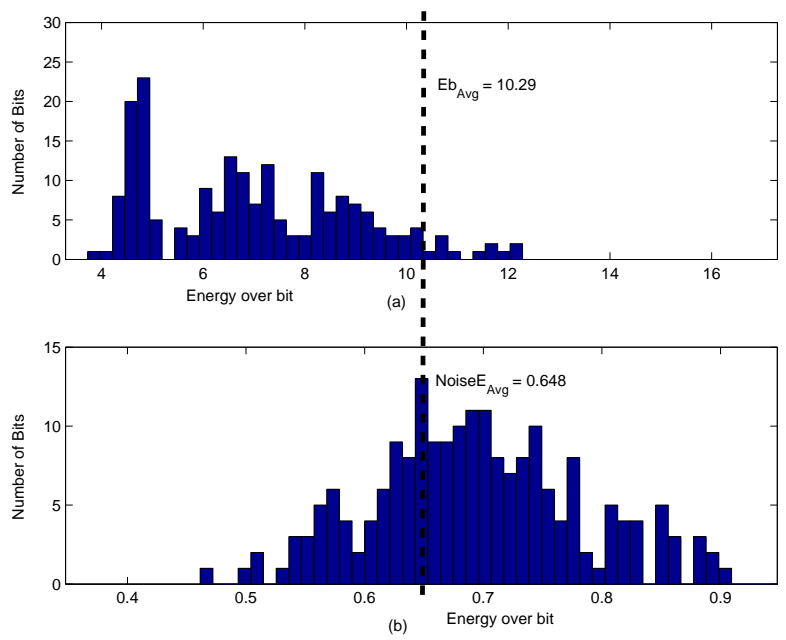

Figure 4: Histograms of (a), bit energy and (b), noise energy for the dual synchronizing response system over 200 errors bits at $\frac{E_{b}}{N_{0}}=29 \mathrm{~dB}$. The average bit energy and average noise energy for all bits is indicated by the dashed line.

a one-bit and the energy of a zero-bit. Let,

$$
E_{\text {diff }}=\left|E_{1}-E_{0}\right|
$$

This energy difference changes for every bit. To improve the systems error performance when subjected to noise, we wish to maximize the average $E_{\text {diff }}$ over all transmitted bits. See Figure 8 for a summary of this parameter optimization work. Thus the goals that $E_{d i f f}$ be maximized, and subject to positive Lyapunov exponents, and leads to a bounded system (trajectories do not escape to infinity), so that we can ensure to work with chaotic oscillators, motivates our specific choice of parameter values.

\section{Frequency Domain Properties and Signal Camouflage}

Since signals are commonly monitored in the frequency domain with a spectrum analyzer, for example in an electronic warfare setting, in this section, we will discuss frequency domain characteristics of our system. As is commonly described for chaotic signal transmission schemes, it can be sold as no more than signal camouflage than encryption.

Using a sweeping spectrum analyzer, we measure in hardware the distribution in the frequency domain. Figure 6 shows that the chaotic signal can be somewhat hidden and not detected in comparison to noise without the use of special techniques. The chaotic signal has the desirable smooth and wide energy distribution property. Analyzing energy over ten sweeps averaged shows the promising property that the spectrum is relatively smooth without distinguishing peaks of energy. This all suggests good camouflage, however it is now well known that these standard chaotic schemes do not make good cryptographic schemes [9].

\section{Chaotic Communication System in Hardware}

There are several difficulties to implement our scheme in hardware. We used two digital signal processors (DSPs) to numerically solve the Lorenz system, the 'C6711 DSP, and we used the 'C6711 DSK transmitter receiver pair, together with two PCM3006 CODEC daughter cards mounted on the DSKs. 
The first of course is timing, which we discussed above by rescaling the equations. A more major hurtle was that our CODECs have a built in high-pass filter fixed to the analog-to-digital converter that cannot be bypassed, since they were designed $t$ be used in audio frequency applications. Frequencies near DC are not significant for audio, but attenuated frequencies are significant for our system for synchronization. Through altering the time scaling $T_{S}$, it is possible to stretch the bandwidth away from $0 \mathrm{~Hz}$, and thus less power is automatically filters. Such significantly improved the synchronization, albeit was never perfect.

Finally, we show a method to amplify difference between transmitter and each of the two receivers. Shown in Figure 8 is a real bit-sequence recovery achieved in hardware shown for one of the two receivers. The transmitter toggles between the two parameter sets based on a message bit from the stream which is $010101 \ldots$, thus dictating the transmitter should toggle parameter values between the two pre-stored values corresponding to each of the two known optimized receivers. E.g., as above, $\beta(0)=4.0$, and $\beta(1)=4.4$, and the other two parameters constant $\sigma=16.0, \rho=45.6$. Rather than instantaneous difference below in blue, cumulative difference over one bit period in red shows good bit differentiation in comparison to a threshold value shown as a dashed line.

\section{Conclusion}

Using a discrete processing approach to explore the benefits of chaos has produced many promising results and has opened up several paths of further research. Discretely generating the chaotic waveforms has both helped to streamline development time and improve upon earlier systems. There are several directions in which the work can be extended. First, a degree of robustness can be added by treating the whole detection step statistically and redundantly. Specifically, consider Figure 8. One cumulative signal (in red below) indicates whether the bit of this receiver should be interpreted, and the Lyapunov exponents of the other system have been tuned by careful choice of parameters so that the other system will desychronize. However in the presence of noise, such will be imperfect. The next step would be to treat the accept or reject oscillator-receiver A or B by statistical hypothesis test, and even to have each in redundant multiple copies to sharpen confidence in the test statistic. It is expected that such will significantly increase system fidelity and will lead to improved BER curves. This will be one direction of our future work.

This work has introduced a new way to consider transmitting information via chaotic carriers. We have discussed system performance as BER curves and we have discussed use by signal camouflage.

\section{References}

[1] L.M. Pecora and T.L. Caroll, "Synchronization in chaotic systems," Phys. Rev. Lett., vol. 64, pp. 821-824, Feb. 1990.

[2] L.M. Pecora et al., "Fundamentals of synchronization in chaotic systems, concepts, and applications," Chaos, vol. 7, pp. 520 - 533, 1997.

[3] K.M. Cuomo, A.V. Oppenheim, and S.H. Strogatz, "Synchronization of Lorenz-Based Chaotic Circuits with Applications to Communications," IEEE Trans. on Circuits and Systems, vol. 40, pp. 626-632, Oct. 1993.

[4] H.K. Khalil, Nonlinear Systems. New York: Prentice Hall. 1996, 2nd ed.

[5] S.H. Strogatz, Nonlinear Dynamics and Chaos. Reading, MA: Addison-Wesley Publishing Co. 1994. 
[6] T.L. Carroll and L.M. Pecora, "Using multiple attractor chaotic systems for communication," Chaos, vol. 9, pp. 445-451, June 1999.

[7] N. Reddell, E. Bollt, T. Welch, "Analysis and Design of a Chaotic Communication System Testbed," proceedings of MILCOM 2002.

[8] Sandri, M. "Numerical Calculation of Lyapunov Exponents." Mathematica J. 6, 78-84, 1996. K. Ramasubramanian, M.S. Sriram, "A comparative study of computation of Lyapunov spectra with different algorithms," chao-dyn/9909029.

[9] A.T. Parker and K.M. Short, "Reconstructing the keystream from a chaotic communication scheme," IEEE Transactions on Circuits and Systems I: Fundamental Theory and Applications. 485 624-630 (2001). 

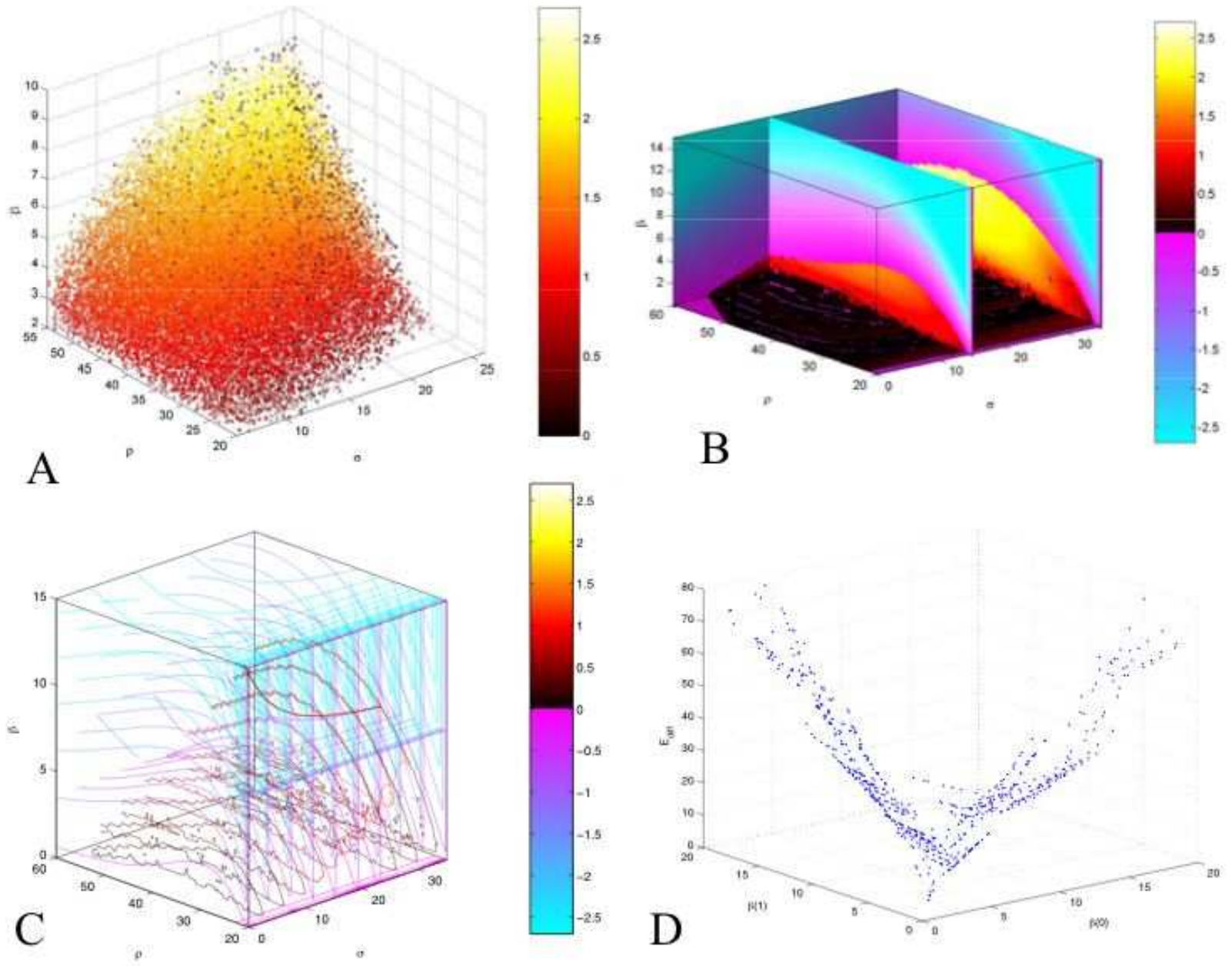

Figure 5: Search for parameter values such that Lyapunov exponents result in maximizing $E_{\text {diff }}$. A) Lyaponov exponents for 100,000 randomly distributed parameter values over parameter space shown. Only the resulting 33,129 positive (and thus chaotic and useful) values are shown. B) Lyapunov exponents are best visualized in parameter space by slices. C) These sliced curves are not in general a continuous function, which makes standard smooth optimization techniques such as gradient descent impossible. D) Dots show bit energy difference $E_{\text {diff }}$ for a pair of $\beta$ value. A search restricted to adjusting only the $\beta$ parameters indicates (but not conclusively due to lack of continuity) that the larger the $\beta$ difference, the higher the associate bit energy. 


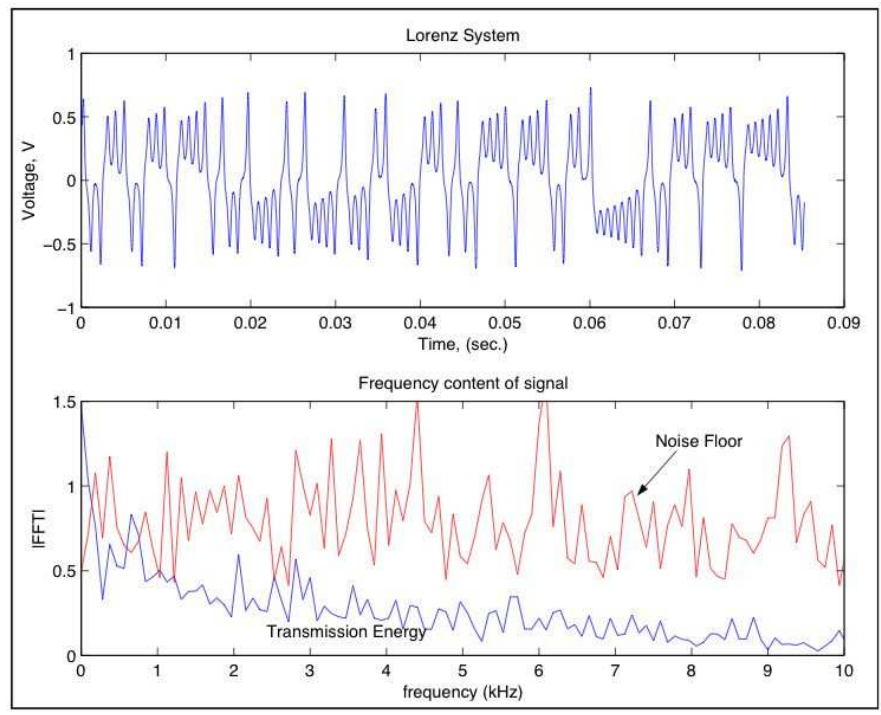

Figure 6: Frequency content of our system transmitter signal indicates energy spread under the noise floor. The ratio of bit energy to noise power spectral density is $\frac{E_{b}}{N_{0}}=25 \mathrm{~dB}$. From Figure 3 , we read that about one in every two thousand bits received are in error.

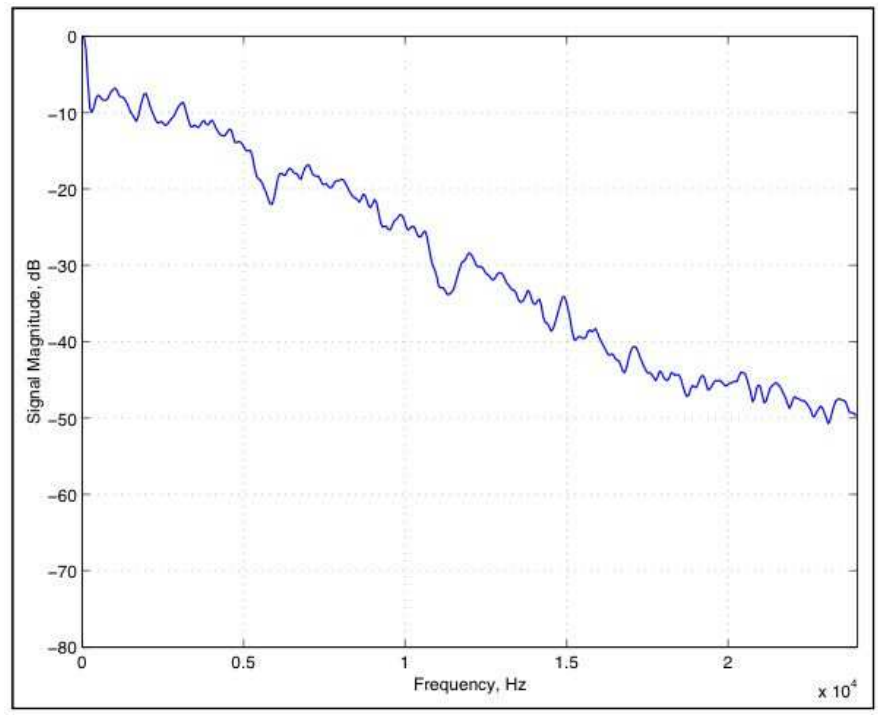

Figure 7: Frequency spectrum of Lorenz system observed on spectrum analyzer with ten video averages. Two notices in the otherwise smooth spectrum remain for longer averages as wel, $(6 \mathrm{kHz}$ and $12 \mathrm{kHz})$. 

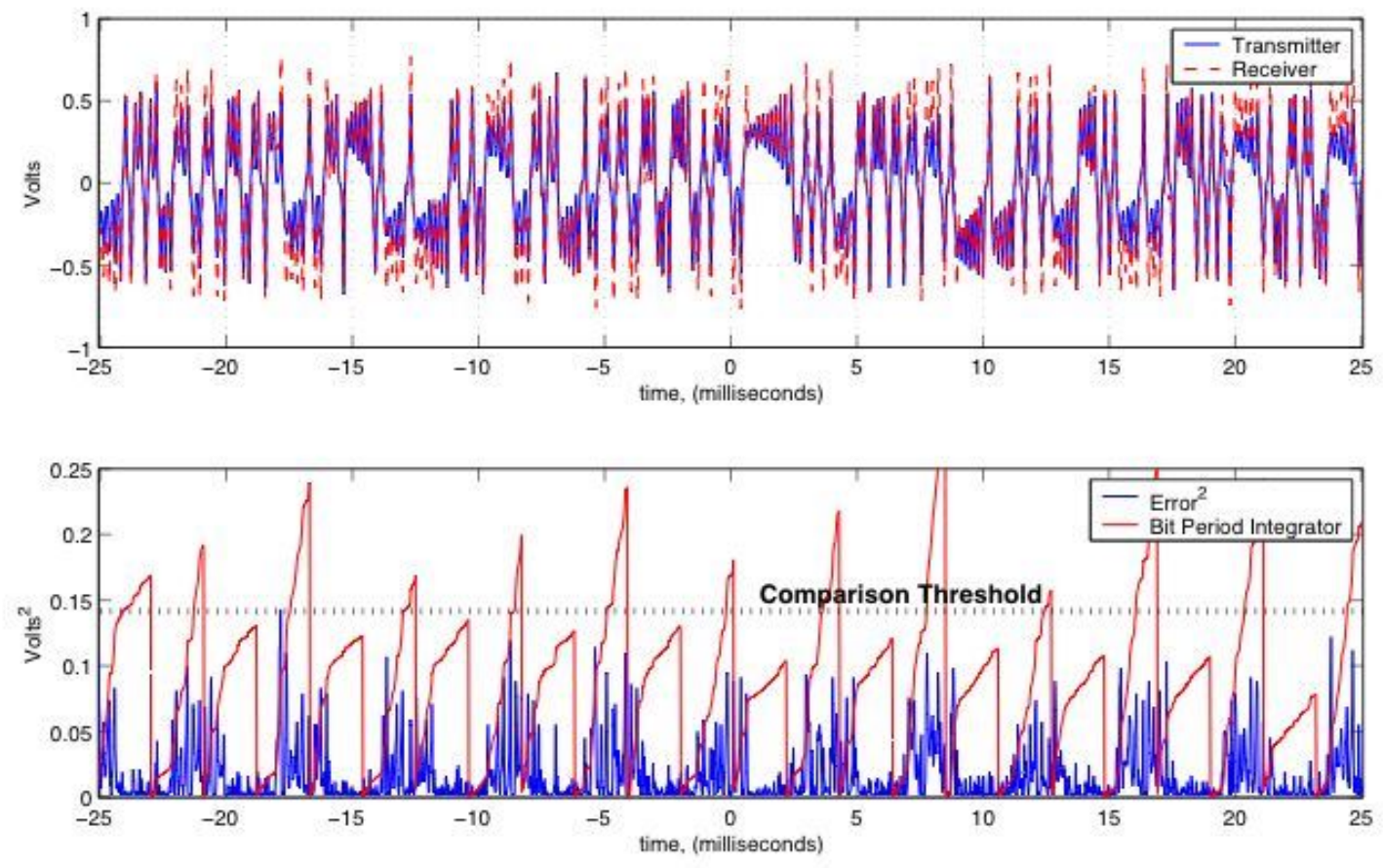

Figure 8: A hardware demonstration of the transmitter sending $010101 \ldots$ by alternating between the two parameter sets $\beta(0)=4.0$, and $\beta(1)=4.4$, and the other two parameters constant $\sigma=16.0$, $\rho=45.6$. over one period, (Above) Transmitting signal in blue and one of two receives in red. (Below) In blue the instantaneous of the receiver system compared to the transmitter signal, and in red the cumulative difference over one bit period. Comparing the cumulative difference to a threshold decides if a one bit should be red corresponding to this of two receivers should be read. 